\title{
Cost-effectiveness analysis of a randomized study of depression treatment options in primary care suggests stepped-care treatment may have economic benefits
}

\author{
Charles Yan $^{1 *}$ (D, Katherine Rittenbach ${ }^{2}$, Sepideh Souri ${ }^{3}$ and Peter H. Silverstone ${ }^{4}$
}

\begin{abstract}
Background: The stepped-care pathway (SCP) model has previously been found to be clinically effective for depressive disorder in some studies, but not all. Several groups have suggested that a stepped-care approach is the most appropriate in primary care. There is relatively little information, however, regarding which specific stepped-care pathway may be best. This analysis aimed to determine cost-effectiveness of a stepped-care pathway for depression in adults in primary care versus standard care (SC), treatment-as-usual (TAU), and online cognitive behavioural therapy (CBT).

Methods: We conducted a randomized trial with 1400 participants and 12-week follow-up to assess the impact of the four treatment options on health-related quality of life and depression severity. Costs for the groups were calculated on the basis of physician, outpatient, and inpatient services using administrative data. We then calculated the incremental cost-effectiveness ratios using this information. Cost-effectiveness acceptability curves and incremental cost-effectiveness scatterplots were created using Monte Carlo simulation with 10,000 replications. A subgroup analysis was conducted for participants who screened as depressed at baseline.

Results: For all participants, TAU was the most expensive followed by CBT, SC, and SCP. QALYs were highest in SCP, followed by SC, CBT, and TAU. In the depressed subgroup, TAU was still the most expensive, followed by SC, SCP, and CBT, while QALYs were still highest in SCP, followed by SC, CBT, and TAU. The cost-effectiveness acceptability curves suggested that SCP had a higher probability for cost-effectiveness than the other three alternatives in all participants. In the depressed subgroup, CBT was associated with the highest probability of cost-effectiveness for a willingness-to-pay cut-off of less than approximately $\$ 50,000$, while SCP was the highest at a cut-off higher than $\$ 50,000$. There is considerable uncertainty around the cost-effectiveness estimates.
\end{abstract}

Conclusions: Our analysis showed that even where there are no clinically significant differences in health outcomes between treatment approaches, there may be economic benefit from implementing the stepped-care model. While more work is required to identify the most clinically effective versions of a stepped-care pathway, our findings suggest that the care pathway may have potential to improve health care system value.

Trial registration: NCT01975207. The trial was prospectively registered on 4 November 2013.

Keywords: Cost-effectiveness, Mental illness, Depression, Primary care, Quality of life

\footnotetext{
* Correspondence: cyan@ihe.ca

'Institute of Health Economics, 1200 - 10405 Jasper Avenue, Edmonton,

Alberta T5J 3N4, Canada

Full list of author information is available at the end of the article
}

(c) The Author(s). 2019 Open Access This article is distributed under the terms of the Creative Commons Attribution 4.0 International License (http://creativecommons.org/licenses/by/4.0/), which permits unrestricted use, distribution, and reproduction in any medium, provided you give appropriate credit to the original author(s) and the source, provide a link to the Creative Commons license, and indicate if changes were made. The Creative Commons Public Domain Dedication waiver (http://creativecommons.org/publicdomain/zero/1.0/) applies to the data made available in this article, unless otherwise stated. 


\section{Background}

Depressive disorder has one of the highest degrees of disease burden in established market economies such as Canada [1-4]. This is due to such factors as incidence (between 10 and 20\% of patients who attend their primary care physicians) [5] and effects such as high rates of unemployment and disability in individuals with major depressive disorder in Canada [6]. Reduction of the considerable disease burden, and its associated economic costs, is a key objective for depression management.

A potential approach to more effective depression management is to universally screen all adults attending primary care and communicate the screening results to the clinic staff for appropriate interventions and/or treatments [7, 8]. When depression has been identified in primary care practice, standard care (SC) can involve a combination of 'watchful waiting', advice, psychotherapy, medication, or referral to a specialist $[9,10]$. Of the psychological treatments available, cognitive behavioural therapy (CBT) is the most commonly used, both in person and electronically via computer $[11,12]$. Given the variety of treatment options, for many years several groups have suggested that a stepped-care treatment approach is the most appropriate in primary care $[13,14]$. The steppedcare pathway (SCP) model usually includes both antidepressant medication and psychosocial interventions, and has previously been found to be clinically effective for depressive disorder in some studies [15-17], but not all $[14,18]$. Furthermore, the implementation of the steppedcare treatment can be both resource and staff intensive, and the specific components of individual programs vary widely $[19,20]$.

There is also relatively little information regarding which specific stepped-care pathway may be best [21]. We have previously reported clinical findings from a randomized, controlled trial (RCT) in adults attending two primary clinics, in which over 1400 patients were screened for depression and randomized into one of four groups [20]: (1) a standard care (SC) control group in which neither patient nor physician knew the results of depression screening; (2) a treatment-as-usual (TAU) group, where physicians were made aware of depression scores, but no guidance regarding treatment was given; (3) an online CBT group in which patients who were depressed were given login information for a well-tested online CBT program; and (4) a stepped-care pathway (SCP). The exception to this randomization was that any participant who scored as high risk for self-harm at any screening time was referred directly to their primary care provider. The stepped-care program we used was developed in Calgary, Alberta, Canada, and was based on existing literature. This program had previously been utilized with 158 patients in an open-label study in five primary care locations during the period 2010-2011 [20, 22].
In our previously reported study [20], the primary clinical outcome was the changes in depression scores, measured by the Patient Health Questionnaire (PHQ-9) [23-25]. The PHQ-9 is a nine-question instrument commonly used for measuring the severity of depression in a primary care setting. Patients' responses to the questions are converted into scores, ranging from 0 to 27 with 0 representing minimal disorder and 27 being the most severe. Patients with a PHQ-9 score greater than 10 are deemed to have moderate to severe depression. Health-related quality of life (HRQoL) was also measured using the EuroQol-5-dimension with a five-level scale (EQ-5D-5 L) [26-28]. All patients were measured at baseline and 12-week post-randomisation, while patients who were depressed at baseline had an additional measurement at six weeks [20]. Interestingly, our results showed no differences in clinical outcomes between any of the four groups [20]. However, we were aware that no previous RCT had examined possible cost implications of the various treatment options, including stepped-care, and had therefore sought to obtain this data a priori, with appropriate ethics committee and subject approval. Therefore, the aim of the current publication is to report on the estimated medical costs of adults who visited their primary care physician and were screened for depression, and to determine whether the stepped-care treatment was cost-effective compared to other approaches both for those who screened positive for depression symptoms and the entire population included in the study.

\section{Method}

\section{Study design and participants}

In this randomised, controlled trial [20], we recruited participants from two primary care clinics in Alberta, Canada. Study groups were block randomized by day (days were randomly assigned to one of the four arms using a random number generator). We assessed participants aged 18 and above, who were able to provide informed consent. The Health Research Ethics Review Board at the University of Alberta approved the study protocol on 30th July 2013. This trial was registered with Clinical Trials database, https://clinicaltrials.gov/ct2/ show/NCT01975207 Identifier: NCT01975207. Details on methods employed in the trial have been published previously [20] and for that reason are only summarized here.

\section{Study arms}

Participants were assigned to one of four arms. In Arm 1 , standard care (SC), the results of PHQ-9 screening were not communicated to the patient or their physician, unless they scored positive for a risk of self-harm. In Arm 2, treatment as usual (TAU), participants who scored greater than 10 on the PHQ-9 had their scores communicated to them and to their physicians for follow 
up without recommendations from the study team. Arm 3 was TAU plus online cognitive behavioural therapy (iCBT). In this arm, participants who scored greater than 10 on the PHQ-9 had their scores communicated to them and to their physicians for follow-up, and patients were provided login information for a free internetbased CBT [29] and encouraged to use it. Participants who scored greater than 10 on the PHQ-9 in Arm 4, known as stepped-care pathway (SCP), had their scores communicated to them and their physicians and were offered a pre-determined treatment as indicated by the depression stepped-care pathway, described in detail elsewhere [20]. Participants in all arms had information collected on their health-related quality of life and symptoms of depression at baseline and week 12 using the EuroQol-5 dimension (EQ-5D-5 L) and the PHQ-9 measure. Those who scored over 10 on the PHQ-9 at baseline also completed the data collection at six weeks, and if at any point any participant scored "at risk" for self-harm, they were referred for urgent treatment.

\section{Data collection}

Patients completed the self-reported Patient Health Questionnaire-9 (PHQ-9), an instrument that can be used to assist in screening and monitoring the severity of depressive symptoms [23], as well as the EQ-5D-5 L at baseline and 12 weeks post-randomization. The EQ$5 \mathrm{D}-5 \mathrm{~L}$ is a standardized measure of health status developed by the EuroQol Group that provides respondents with a descriptive system to classify their health status based on five dimensions: mobility, self-care, usual activities, pain/discomfort, and anxiety/depression [27, 30]. This measurement provides a utility score for each of the EQ5D-5 L health states between values of 0 and 1 , with 1 representing the best (perfect) health state and 0 the worst (death) state. The analysis was performed from the perspective of health care payer, and the costs and resource utilization included in the analysis were for physician, outpatient, and inpatient services. Physician services include all activities performed in primary, outpatient, and inpatient care settings. Inpatient and outpatient costs cover all activities other than physician service in these settings. Examples of these costs include salaries, drugs, medical and surgical supplies, administration, and support services. We collected data during the periods of 12 weeks pre-randomization, 12 weeks post-randomization, and from 12 weeks to 1-year post-randomization. While some data were also collected in depressed patients at week six (approximately $20 \%$ of the total sample), it is not included in the present analysis. Data on health care system usage were retrieved from health administrative databases that provide individual patient information such as age, gender, cost, diagnostics, and service date. Since there is a single health care service in the province of Alberta, this information comprised all relevant health spending for the study patients with the exception of private psychological services, which we are unable to include in the analysis. In other words, the analysis included direct medical costs only, while other societal costs, such as productivity losses and transportation, were not included.

\section{Statistical analysis}

The analysis followed the guidelines set by the International Society for Pharmacoeconomics and Outcomes Research (ISPOR) Good Research Practices Task Force [31, 32]. An intention-to-treat (ITT) approach that considered all participants allocated to each intervention arm was applied for the analysis; this approach was utilized to avoid bias [31, 33]. Missing data were handled using multiple imputation, which reflects inherent uncertainty when replacing missing data [31, 32, 34]. The data from each of the four intervention arms were further separated into a 'depression' subgroup of participants, who scored greater than 10 on the PHQ-9 at baseline. The analysis focuses on the depression subgroup as this is the group that may have received clinical benefit from the treatment options, since those who did not have symptoms of depression had no need for the interventions. An overall PHQ-9/EQ-5D-5 L score was estimated for each intervention arm. Imbalances in baseline PHQ-9 and EQ5D-5 L scores were accounted for using ordinary least squares (OLS) regression in estimating their values at 12week post-randomization. The difference between intervention arms was tested using a one-way ANOVA test, the difference between each pair of intervention arms was tested using a pairwise comparison of mean, and the difference between baseline and 12-week post-randomisation for each intervention arm was tested using paired t-test for these scores [35].

Data investigation indicated unusually higher costs for some participants, known as outliers, compared with the average cost for each study group. Outliers skew average costs and are unlikely to represent the true average expenditure for healthcare services [36, 37]. The outliers in each period and intervention arm were trimmed. We used a traditional univariate boxplot to trim physician and outpatient costs, with cost data beyond 1.5 times the interquartile range (IQR) being excluded. We did not trim outliers for inpatient costs because of the limited available data, as there were only a few participants admitted to hospital. A generalized linear model (GLM) was applied to adjust for imbalance in baseline characteristics such as age, gender, and PHQ-9 scores in estimating costs at 12-week and one-year post-randomization. The GLM extends the linear modelling approach to data that are not normally distributed. We used a gamma distribution as the family suitable when fitting skewed healthcare cost data [38]. The link function used in GLM specifies how the mean of the dependent variable depends on the 
predictors. In the analysis we used identity link function, implying a linear relation between the cost and predictors. Predicted cost values from the GLM were used to represent the costs for each period and each intervention arm. Discounting was not required, as the study time horizon was just one year. All costs were adjusted to a standard price year of 2017, using the Alberta Consumer Price Index (CPI).

We compared the costs and cost-effectiveness of the intervention arms at one year to capture the economic effect of the alternatives. We used quality-adjusted lifeyears (QALYs) as the primary outcome to measure health effectiveness over 12 months. We calculated QALYs as the area under the curve defined by the EQ5D-5 L utility scores at baseline and one year. The RCT did not collect the EQ-5D-5 L data after 12-week post randomisation, and we therefore assumed the utility scores over the time point remained unchanged. This assumption implies that treatment effect is maximized in the first 12 weeks. We conducted a sensitivity analysis on two different scenarios to test the impact of this assumption.

Health care utilization data for participants in the RCT indicated that, during the year after randomization, a participant received either no medical services or a combination of physician, outpatient, and/or inpatient services. As shown in Table 1, approximately 1 to $2 \%$ of participants did not receive any medical services; 40 to $45 \%$ had physician visits only; 40 to $48 \%$ had a combination of physician visits plus outpatient visits; 0 to $1.7 \%$ had a combination of physician plus inpatient admission; and 9 to 17\% received medical services from all the sectors (physician visits, outpatient visits, and inpatient admission).

The SCP group had a small likelihood of using expensive inpatient services in this study, which significantly impacted overall spending observed during the trial. In order to verify that the results of this analysis were not biased due to this decrease in inpatient spending, we developed a decision tree model to capture the cost of each combination of services for participants. This model splits participants into one of the five combinations reported in Table 1 and assigns the probabilities of service use to each group. We used the decision tree in the base case analysis, and because the likelihood of inpatient use is the main component driving difference in cost-effectiveness, we conducted a one-way sensitivity analysis to assess the impact of changing the likelihood.

The incremental cost-effectiveness ratio (ICER) was calculated as the ratio of differences in mean costs and mean number of QALYs [39]. The differences in means were assessed using Monte Carlo Simulation, a mathematical technique to address the uncertainty of the cost effectiveness. The input cost and effectiveness data were repeatedly modelled for 10,000 iterations on the basis of known probability distributions for generating the outcomes [31, 40-42]. Based on results from the Monte Carlo simulations, we constructed the cost-effectiveness acceptability curves and incremental cost-effectiveness scatterplots to help the understanding of the uncertainty of the ICER [39, 43]. In addition to the analysis performed on the overall participants, the cost-effectiveness analysis was performed in a subgroup of participants who scored greater than 10 on the PHQ-9 at baseline $[31,44]$. The statistical analysis was performed with the Stata software package (Release 13.1) for Windows, and decision analytic modelling analysis was performed with TreeAge Pro 2015 software (TreeAge Software, Inc).

\section{Results}

We recruited 1400 participants between November 2013 to December 2014, randomly allocating 412 (29\%) to SC, 397 (28\%) to TAU, 415 (29\%) to ICBT, and 183 (17\%) to $\mathrm{SCP}$. Note that the numbers in each arm were not

Table 1 Number of participants receiving physician, outpatient, and/or inpatient services during first year after randomization

\begin{tabular}{|c|c|c|c|c|c|c|}
\hline Arm & No services & Physician only & Physician + outpatient & Physician + inpatient & Physician + outpatient + inpatient & Total \\
\hline \multicolumn{7}{|c|}{ All participants } \\
\hline SC & $4(1.0 \%)$ & $179(43.4 \%)$ & $176(41.99 \%)$ & $3(0.73 \%)$ & $53(12.9 \%)$ & 412 \\
\hline TAU & $8(2.0 \%)$ & $160(40.3 \%)$ & $160(39.80 \%)$ & $2(0.5 \%)$ & $69(17.4 \%)$ & 397 \\
\hline ICBT & $4(1.0 \%)$ & $188(45.3 \%)$ & $164(37.83 \%)$ & $7(1.69 \%)$ & $59(14.2 \%)$ & 415 \\
\hline SCP & $3(1.6 \%)$ & $75(41.0 \%)$ & $88(48.08 \%)$ & na & $17(9.3 \%)$ & 183 \\
\hline All & $19(1.4 \%)$ & $602(42.8 \%)$ & $588(40.94 \%)$ & $12(0.85 \%)$ & $198(14.1 \%)$ & 1407 \\
\hline \multicolumn{7}{|c|}{ Depressed subgroup } \\
\hline SC & $0(0 \%)$ & $21(37.5 \%)$ & $28(50 \%)$ & na & $7(12.5 \%)$ & 56 \\
\hline TAU & $2(3 \%)$ & $2131.8(\%)$ & $35(53 \%)$ & na & $8(12.1 \%)$ & 66 \\
\hline ICBT & $0(0 \%)$ & $22(44 \%)$ & $23(46 \%)$ & na & $5(10 \%)$ & 50 \\
\hline SCP & $0(0 \%)$ & $12(35.3 \%)$ & 18 (52.9\%) & na & $4(11.8 \%)$ & 34 \\
\hline All & $2(1 \%)$ & 76 (36.9\%) & 104 (50.5\%) & na & $24(11.7 \%)$ & 206 \\
\hline
\end{tabular}


balanced, as not all clinics were able to offer the stepped-care treatment. Of the 1400 total subjects, 206 participants scored greater than 10 on the PHQ-9 at baseline (referred to as "depressed" henceforth), with 56 (27\%), 66 (32\%), 50 (24\%), and 34 (17\%) being depressed in Arms 1 to 4, respectively. Table 2 presents baseline demographics and outcomes measured in terms of PHQ-9 and EQ-5D-5 L. The mean (s.d.) age was 47 (17) years in all participants and 45 (15) years in depressed ones, and the majority was female $(73 \%$ in all participants and $74 \%$ in depressed ones). More data are available in our Additional file 1.

We found statistically significant improvement in PHQ-9 and EQ-5D-5 L from baseline to 12-week post randomisation in all arms (Table 2). In all participants, the mean change between baseline and 12-week was 0.72 (95\% CI 0.61-0.82) in PHQ-9 and 0.024 (95\% CI 0.021-0.027) in EQ-5D-5 L. As expected, the amount of improvement was greater in depressed participants, for whom the mean change was 4.8 (95\% CI 4.58-5.02) in PHQ-9 and 0.103 (95\% CI 0.092-0.115) in EQ-5D-5 L. There was no evidence of superiority in clinical effectiveness between arms.
For the economic analyses, we used the decision tree model to estimate the cost for inpatient, outpatient, and physician services for all participants and the subgroup of depressed participants, during the one-year period post-randomization (Table 3). The costs in participants who screened as depressed at baseline were higher compared with all participants over the year. We also compared inpatient, outpatient, and physician costs for depressed and non-depressed patients over three periods: 12 weeks pre-randomization (prior to screening), 12 weeks postrandomization (treatment duration), and 12 weeks to one-year post-randomization (Table 4). We found evidence of difference in costs for all the service sectors and over all the time periods with one exception. That one exception is inpatient cost from 12 weeks to oneyear, where the cost difference was not statistically significant. As mentioned, few participants were admitted to inpatient care during the study.

The results of the cost-effectiveness analysis (Table 5) show that for all participants, TAU was associated with the highest total cost, followed by iCBT, SC, and SCP with SCP being the least costly alternative. Further, QALY was highest in SCP, followed by SC, iCBT, and

Table 2 Description of the sample

\begin{tabular}{|c|c|c|c|c|c|}
\hline \multicolumn{6}{|l|}{ All participants } \\
\hline & SC $(n=412)^{\S}$ & TAU $(n=397)$ & ICBT $(n=415)$ & $\operatorname{SCP}(n=183)$ & All $(n=1407)$ \\
\hline Age (s.d.) & $46.9(17.1)$ & $45.5(16.3)$ & $46.6(16.8)$ & $52.3(17.6)$ & $47.1(17.0)$ \\
\hline Female (\%) & $75 \%$ & $78 \%$ & $70 \%$ & $61 \%$ & $73 \%$ \\
\hline PHQ-9 (s.d.), baseline & $4.55(4.95)$ & $4.98(5.19)$ & $4.2(4.61)$ & $4.86(5.54)$ & $4.61(5.01)$ \\
\hline PHQ-9 (s.d.), 12-week & $3.85(2.94)$ & $4.12(3.09)$ & $3.65(2.74)$ & $4.04(3.29)$ & $3.89(2.98)$ \\
\hline within group difference $(95 \% \mathrm{Cl})^{*}$ & 0.69 (0.50 to 0.98$)$ & 0.88 (0.67 to 1.08$)$ & 0.55 (0.37 to 0.73$)$ & 0.82 (0.49 to 1.15$)$ & 0.72 (0.61 to 0.82) \\
\hline EQ-5D-5 L (s.d.), baseline & $0.86(0.11)$ & $0.84(0.14)$ & $0.86(0.12)$ & $0.86(0.13)$ & $0.86(0.12)$ \\
\hline EQ-5D-5 L (s.d.), 12-week & $0.88(0.06)$ & $0.87(0.08)$ & $0.88(0.07)$ & $0.88(0.08)$ & $0.88(0.07)$ \\
\hline within group difference $(95 \% \mathrm{Cl})^{* *}$ & $0.022(0.017$ to 0.026$)$ & 0.03 (0.024 to 0.035$)$ & 0.022 (0.017 to 0.027$)$ & 0.022 (0.014 to 0.03 ) & 0.024 (0.021 to 0.027$)$ \\
\hline \multicolumn{6}{|c|}{ Depressed participants (PHQ-9 > 10 at baseline) } \\
\hline & $\mathrm{SC}(n=56)$ & TAU $(n=66)$ & ICBT $(n=50)$ & $\operatorname{SCP}(n=34)$ & All $(n=206)$ \\
\hline Age (s.d.) & $44.7(16.3)$ & $44.8(14.2)$ & $42.2(13.8)$ & $49.0(16.4)$ & $44.8(15.1)$ \\
\hline Female (\%) & $80 \%$ & $70 \%$ & $70 \%$ & $74 \%$ & $74 \%$ \\
\hline PHQ-9 (s.d.), baseline & $14.73(4.17)$ & $14.83(3.91)$ & $14.28(3.76)$ & $14.85(3.84)$ & $14.67(3.91)$ \\
\hline PHQ-9 (s.d.), 12-week & $9.9(2.48)$ & $9.94(2.31)$ & $9.64(2.24)$ & $9.98(2.28)$ & $9.86(2.32)$ \\
\hline within group difference $(95 \% \mathrm{Cl})^{*}$ & 4.83 (4.37 to 5.28$)$ & 4.85 (4.46 to 5.24$)$ & 4.64 (4.21 to 5.08$)$ & 4.88 (4.33 to 5.42$)$ & 4.8 (4.58 to 5.02$)$ \\
\hline EQ-5D-5 L (s.d.), baseline & $0.68(0.18)$ & $0.63(0.2)$ & $0.67(0.22)$ & $0.68(0.21)$ & $0.66(0.2)$ \\
\hline EQ-5D-5 L (s.d.), 12-week & $0.78(0.1)$ & $0.74(0.12)$ & $0.77(0.13)$ & $0.78(0.12)$ & $0.76(0.12)$ \\
\hline within group difference $(95 \% \mathrm{Cl})^{* *}$ & 0.095 (0.075 to 0.115$)$ & 0.118 (0.096 to 0.139$)$ & $0.1(0.075$ to 0.126$)$ & 0.094 (0.064 to 0.125$)$ & 0.103 (0.092 to 0.115$)$ \\
\hline
\end{tabular}


Table 3 Costs for physician, outpatient, and inpatient services for one year after randomization

\begin{tabular}{|c|c|c|c|c|}
\hline \multirow[t]{2}{*}{ Study arm } & \multicolumn{2}{|c|}{ Overall } & \multicolumn{2}{|c|}{ Depressed subgroup } \\
\hline & $n$ & mean (s.d.) [range] & $n$ & mean (s.d.) [range] \\
\hline \multicolumn{5}{|c|}{ Inpatient Stay } \\
\hline SC & 56 & 8472.98 (4916) [2242 to 23,468$]$ & 7 & $11,953.99$ (4257) [7091 to 16,843$]$ \\
\hline TAU & 70 & 8158.80 (5819) [1132 to 32,349] & 7 & $14,771.99$ (6465) [5837 to 27,309$]$ \\
\hline ICBT & 66 & 8477.81 (5340) [2119 to 33,459] & 5 & 9357.69 (2698) [6232 to 13,415$]$ \\
\hline SCP & 17 & $10,670.27$ (3994) $[4060$ to 16,724$]$ & 4 & $10,820.99$ (2660) [8528 to 14,574$]$ \\
\hline All & 209 & 8548.00 (5309) [1132 to 33,459$]$ & 23 & $12,050.19$ (4787) [5837 to 27,309$]$ \\
\hline \multicolumn{5}{|l|}{ Outpatient } \\
\hline SC & 198 & 1036.58 (386) [535 to 2123] & 33 & 1256.53 (498) [593 to 2123] \\
\hline TAU & 200 & 1002.32 (392) [497 to 2127] & 38 & 1188.6 (428) [637 to 2127] \\
\hline ICBT & 193 & 945.89 (340) [480 to 1711] & 24 & 1101.8 (420) [488 to 1711$]$ \\
\hline SCP & 90 & 1144.49 (611) [103 to 2993] & 21 & $1800.39(648)[641$ to 2993$]$ \\
\hline All & 681 & 1015.07 (416) [103 to 2993] & 116 & 1300.72 (543) [488 to 2993] \\
\hline \multicolumn{5}{|l|}{ Physician } \\
\hline SC & 395 & 683.75 (241) [89 to 1300] & 52 & 802.56 (285) [200 to 1300] \\
\hline TAU & 375 & 758.17 (269) [250 to 1534] & 60 & 996.41 (326) [341 to 1534] \\
\hline ICBT & 394 & 667.49 (214) [160 to 1045$]$ & 48 & 787.08 (216) [160 to 1045$]$ \\
\hline SCP & 172 & 559 (207) [173 to 901] & 31 & 633.6 (648) [234 to 901] \\
\hline All & 1336 & 683.78 (245) [89 to 1534$]$ & 191 & 832.14 (298) [160 to 1534$]$ \\
\hline
\end{tabular}

TAU. In those who screened as depressed at baseline, TAU was still the most expensive, followed by SC, SCP, and $\mathrm{iCBT}$ with $\mathrm{iCBT}$ being the least costly alternative rather than SCP. However, QALY was still highest in SCP, followed by SC, iCBT, and TAU. The cost-effectiveness acceptability curves revealed that, for the entire cohort, SCP was associated with the highest probability of being cost-effective over a range of willingness-topay from 0 to $\$ 200,000$ (Fig. 1). In the subgroup of depressed participants, ICBT was associated with the highest probability of cost-effectiveness for a willingness-to-pay from 0 to approximately $\$ 50,000$, while SCP was highest at a willingness-to-pay greater than $\$ 50,000$ (Fig. 2).

Table 4 Inpatients, outpatient and physician costs for depressed and non-depressed patients ${ }^{\S}$

\begin{tabular}{|c|c|c|c|c|}
\hline \multirow[t]{2}{*}{ Period } & \multicolumn{2}{|c|}{ Depressed patients } & \multicolumn{2}{|c|}{ Non-depressed patients } \\
\hline & $\mathrm{n}$ & Mean (s.d.) [Range] & $\mathrm{n}$ & Mean (s.d.) [Range] \\
\hline \multicolumn{5}{|l|}{ Inpatient } \\
\hline 12-Week Prior** & 10 & $10,010.70(2812)[5720$ to 13,469$]$ & 51 & 8517.04 (3913) [3338 to 16,933$]$ \\
\hline 12-Week Post* & 7 & 9544.90 (2272) [6216 to 11,890$]$ & 76 & 5664.30 (2229) [2768 to 13,550$]$ \\
\hline One year** & 17 & 9441.57 (3339) [5025 to 15,311$]$ & 116 & 9429.63 (4577) [2968 to 19,157$]$ \\
\hline \multicolumn{5}{|l|}{ Outpatient } \\
\hline 12-Week Prior* & 57 & 727.89 (195) [141 to 1057] & 245 & $651.66(105)[147$ to 774$]$ \\
\hline 12-Week Post* & 67 & 732.77 (136) [488 to 1055] & 265 & $571.43(66)[355$ to 765$]$ \\
\hline One year* & 91 & 1118.55 (242) [641 to 1938] & 441 & 881.98 (156) [103 to 1371$]$ \\
\hline \multicolumn{5}{|l|}{ Physician } \\
\hline 12-Week Prior* & 147 & 282.32 (52) [110 to 401] & 734 & 228.04 (35) [56 to 322] \\
\hline 12-Week Post* & 178 & 346.92 (73) [151 to 510] & 997 & 260.22 (44) [89 to 374$]$ \\
\hline One year* & 157 & 619.02 (149) [251 to 1024$]$ & 964 & 513.65 (89) [213 to 781$]$ \\
\hline
\end{tabular}

$\S:$ Depressed and non-depressed patients were those who scored greater than 10 and less than 10 on PHQ-9 at baseline, respectively

*: One-way ANOVA test: statistically significant difference between depressed and non-depressed patients $(p<0.001)$

**: One-way ANOVA test: no statistically significant difference between depressed and non-depressed patients $(p>0.25)$

s.d. Standard Deviation, Range: Minimum to Maximum 
Table 5 Mean cost and QALY per participant

\begin{tabular}{|c|c|c|c|c|}
\hline Sector & SC & TAU & ICBT & SCP \\
\hline \multicolumn{5}{|l|}{ All participants } \\
\hline Physician Mean (s.d.) & $\$ 678(239)$ & $\$ 744(263)$ & $\$ 661(213)$ & $\$ 548(205)$ \\
\hline Outpatient Mean (s.d.) & $\$ 572(212)$ & $\$ 575(228)$ & $\$ 490(177)$ & $\$ 653(351)$ \\
\hline Inpatient Mean (s.d.) & $\$ 1096(630)$ & $\$ 1421(1006)$ & $\$ 1202(758)$ & $\$ 990(372)$ \\
\hline Total (s.d.) & $\$ 2346(655)$ & $\$ 2740(968)$ & $\$ 2353(767)$ & $\$ 2191(539)$ \\
\hline Cost Difference (95\% CI) (SCP vs. others) & $-\$ 155(-173---138)$ & $-\$ 549(-585---514)$ & $-\$ 161(-200---123)$ & - \\
\hline QALY (s.d.) & $0.881(0.003)$ & $0.870(0.004)$ & $0.881(0.003)$ & $0.882(0.005)$ \\
\hline QALY Difference (95\% CI) (SCP vs. others) & $0.0001(0--0.0004)$ & $0.0124(0.012--0.013)$ & $0.0003(0--0.0008)$ & - \\
\hline \multicolumn{5}{|l|}{ Depressed participants } \\
\hline Physician Mean (s.d.) & $\$ 794(281)$ & $\$ 966(316)$ & $\$ 784(217)$ & $\$ 634(210)$ \\
\hline Outpatient Mean (s.d.) & $\$ 787(314)$ & $\$ 770(278)$ & $\$ 618(234)$ & $\$ 1163(416)$ \\
\hline Inpatient Mean (s.d.) & $\$ 1497(535)$ & $\$ 1779(780)$ & $\$ 1119(321)$ & $\$ 1279(315)$ \\
\hline Total (s.d.) & $\$ 3078(677)$ & $\$ 3516(890)$ & $\$ 2522(455)$ & $\$ 3075(565)$ \\
\hline Cost Difference (95\% Cl) (SCP vs. others)) & $-\$ 3(-20-14)$ & $-\$ 440(-472---409)$ & $\$ 554(525--582)$ & - \\
\hline QALY (s.d.) & $0.766(0.014)$ & $0.730(0.014)$ & $0.758(0.017)$ & $0.767(0.021)$ \\
\hline QALY Difference (95\% CI) (SCP vs. others) & $0.0013(0--0.0025)$ & $0.037(0.035--0.039)$ & $0.0097(0.008--0.012)$ & - \\
\hline
\end{tabular}

The scatterplot of the simulated costs and QALYs shows pairs of values from the 10,000 simulations on the incremental cost-QALY plane between SCP versus iCBT. Note that scatterplots comparing with $\mathrm{SC}$ and TAU were not presented as they were both dominated by iCBT and SCP and were therefore excluded for further consideration. In all participants, there were 55\% of scatter points falling under the $\$ 50,000$ threshold line where SCP was deemed to be cost-effective; in depressed participants, there were $52 \%$ of scatter points under the line (Figs. 3 and 4). The scatterplots of TAU compared with $\mathrm{SC}$ are available in the Additional file 1.

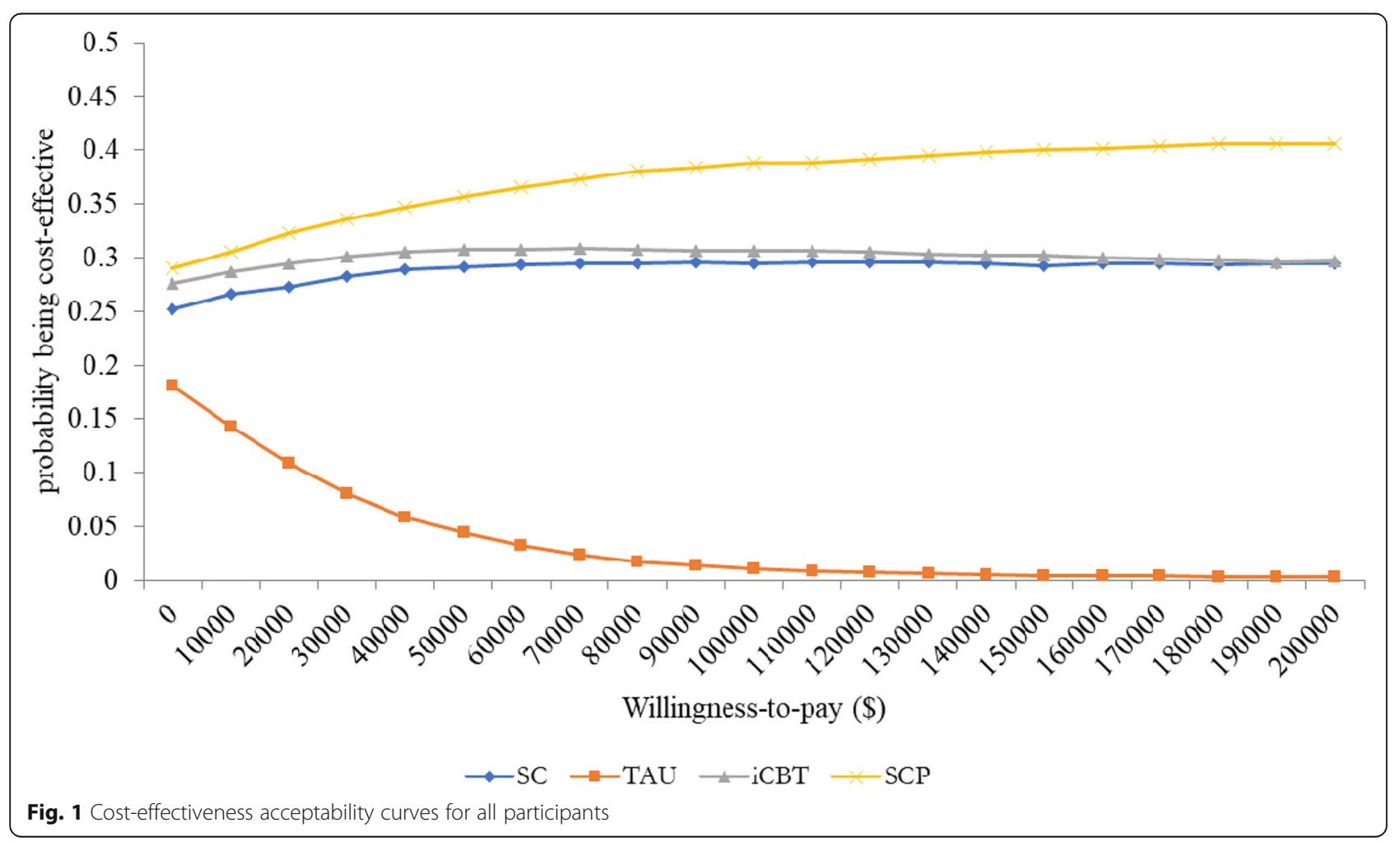




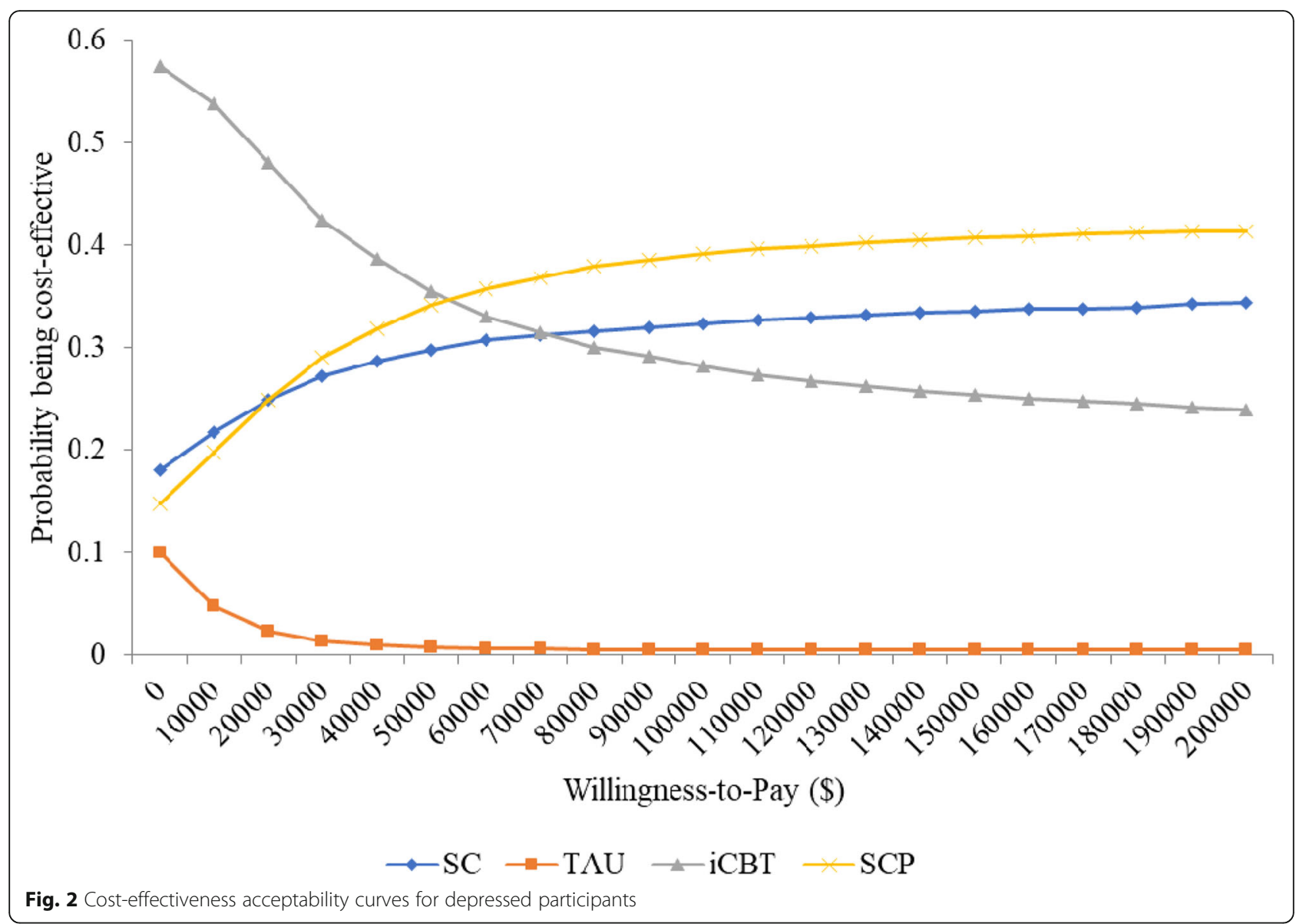

We expected that the combination of physician, outpatient, and/or inpatient services that a patient received (Table 1) would have a large impact on the cost-effectiveness results, and therefore conducted a sensitivity analysis on a hypothesised scenario that assumed the four intervention arms had identical distribution of patients receiving each combination of medical services (See the rows named "All" in Table 1). In this sensitivity analysis and for all participants, ICBT replaced SCP and became dominating in that it was associated with the highest probability of being cost-effective (Fig. 5). This unsurprising finding was mainly driven by the large increase in the number of theoretical participants receiving physician plus outpatient plus inpatient services in SCP. According to our assumption, this portion in SCP jumped from $9.3 \%$ at base-case analysis to $14 \%$ in the sensitivity analysis (Table 1). In the subgroup of depressed participants, the results of the sensitivity analysis remained close to the base-case analysis with a slight move leftward of the willingness-to-pay cut-off threshold from approximately $\$ 50,000$ at base-case analysis to $\$ 40,000$ per QALY (Fig. 6). This close result between base case analysis and sensitivity analysis was anticipated, given that no substantial changes appeared in this portion (from $12 \%$ for ICBT and $11.8 \%$ for SCP at base-case analysis to $11.7 \%$ at the sensitivity analysis; see Table 1).

To test the assumption that the treatment effect remained unchanged after the 12-week mark for one year, we conducted a sensitivity analysis by assuming (1) the treatment effect gradually reduced to baseline values from 12- to 52-week and (2) the treatment effect immediately reduced to baseline values at 12 -week. When giving consideration to the uncertainty in the parameter estimates, we found that the cost-effectiveness results were barely sensitive to the assumptions. The results of the sensitivity analysis are reported in the Additional file 1 in Figures A.3 and A.6.

\section{Discussion}

Our study found that the stepped-care pathway (SCP) for depression was neither superior nor inferior to standard care (SC), treatment as usual (TAU), or treatment as usual plus online-based cognitive behaviour therapy (iCBT) in terms of depression symptom reduction and health-related quality of life (HRQoL) [20]. Interestingly, the present cost-effectiveness analysis suggested SCP is more 


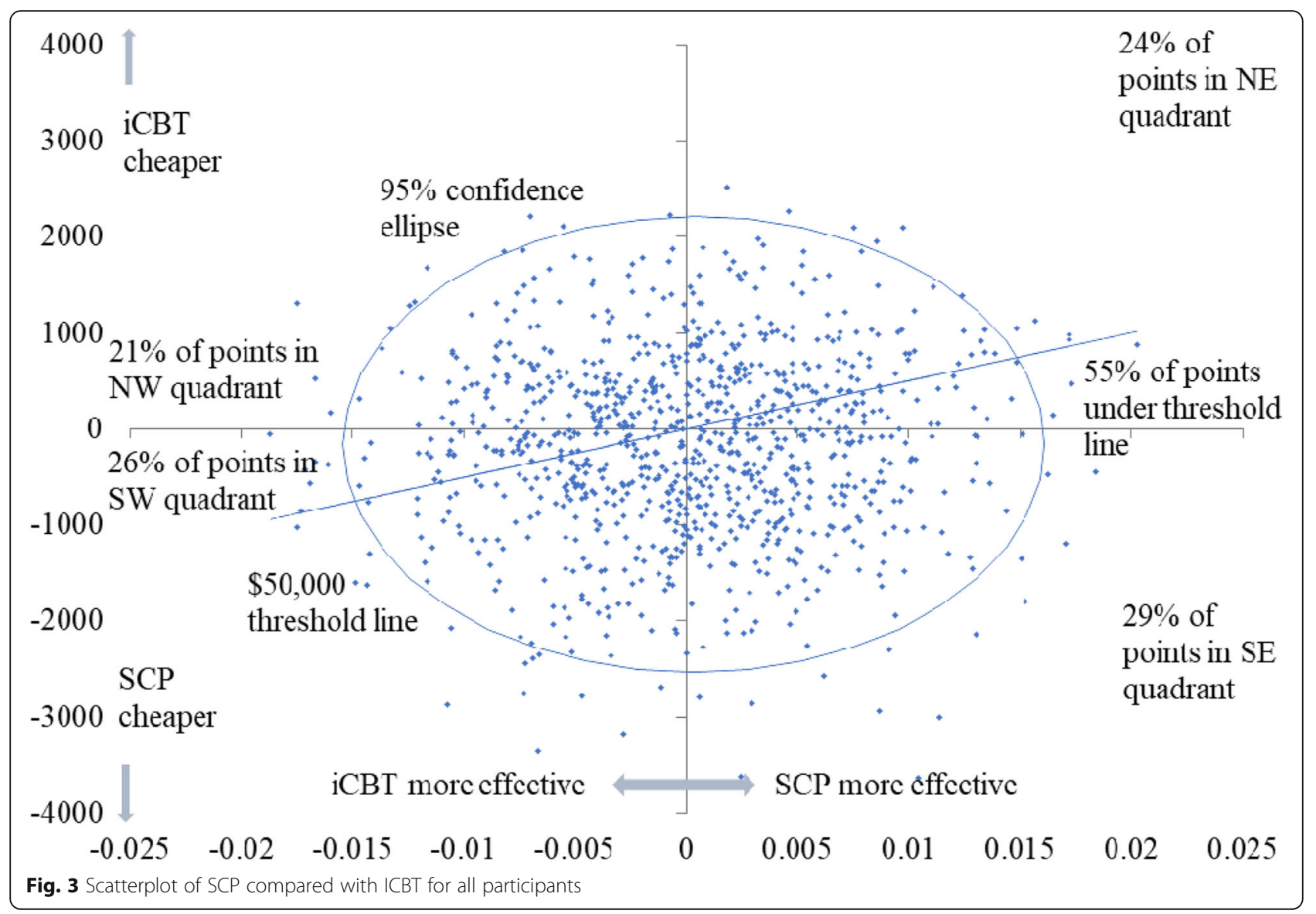

cost-effective than the other alternatives regardless of how much a decision-maker is willing to pay for a QALY gain. Furthermore, in the subgroup analysis of the depressed participants, SCP is cost-effective against the commonly used willingness-to-pay thresholds of $\$ 50,000$.

It should be noted that the threshold of $\$ 50,000$ per QALY is a conservative decision rule. The threshold of $\$ 50,000$ per QALY has been argued to lack theoretical and empirical justification $[45,46]$. Furthermore, it has been suggested that this $\$ 50,000$ sum has not been adjusted for inflation and changes in increasing healthcare expenditures since its debut in the 1990s [47]. Indeed, others have recommended the use of a willingness-topay threshold of $\$ 50,000, \$ 100,000$, and $\$ 200,000$ per QALY [45]. If such higher thresholds were used in the current study, then our cost-effectiveness results would be more strongly in favour of SCP.

Interpretation of our results should take into account uncertainty around the cost-effectiveness estimates. Our scatterplots and cost-effectiveness acceptability curves suggested a large degree of uncertainty, making the decision harder when choosing an alternative. While the number of recruited participants was quite high (1400), the final numbers in each arm that had symptoms of depression became relatively small. This lower incidence may contribute considerably to the uncertainty, since a small number is in general associated with larger standard errors and reduces statistical power. We therefore suggest further work with a larger sample size to enhance confidence in the selection between treatment alternatives.

We modelled care costs and considered the combination of physician, outpatient, and inpatient services. Hospital stays are much more expensive than outpatient and physician visits. In our data, hospital stays were approximately eight times the cost of outpatient services and 12 times the cost of physician services, implying hospital stays make up a large percentage of the total cost. Intuitively, if a treatment approach can reduce the likelihood of admittance to a hospital and/or an outpatient visit, then costs will be reduced. We therefore included the distribution of patients receiving each type of care. Our finding that SCP resulted in similar QALY gained but at a financial savings may be driven by a relatively small portion of patients receiving relatively expensive hospital stays. Our sensitivity analysis confirmed this aspect of the study and revealed the cost-effectiveness results were sensitive to the number of patients admitted to hospital.

Among the published cost-effectiveness studies for screening and treatment of depression in primary care 


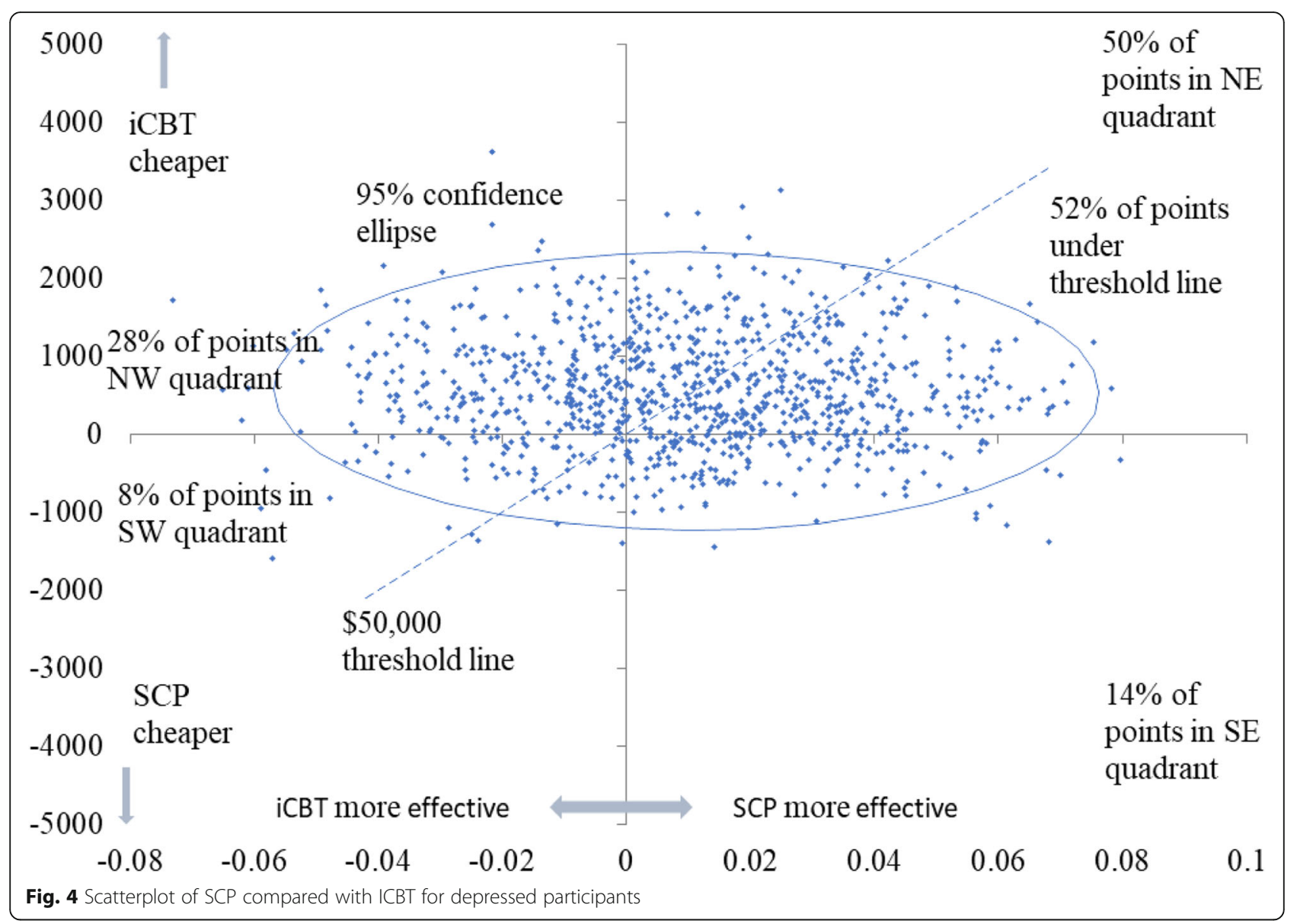

([48-51]), we are not aware of any that simultaneously evaluated the same four intervention alternatives. However, we are aware of studies evaluating the cost-effectiveness of stepped-care pathways (SCP) compared with treatment as usual (TAU), and these studies have reported results consistent with our findings. Thus, Grochtdreis et al. [51] systematically reviewed 19 cost-effectiveness studies that compared stepped-care pathways with treatment as usual for depression in primary care, and found stepped-care pathways were generally more cost-effective. In addition, an economic evaluation in UK primary care [50] demonstrated that improving access to psychological therapies in a stepped approach was cost-effective.

Despite the use of universal screening and an RCT to control some of the biases inherent in such research, we are aware that the present study has limitations. The first is a concern regarding methodology as discussed in our previously published study [20]. Participants were only recruited in two primary care clinics, and while both were trained on the stepped-care pathway, only one of these clinics actively recruited into the steppedcare pathway group. This limited recruitment led to a much smaller sample size in this particular group than the others, and it also had the lowest retention rates for the study. Therefore, clinic differences could, in part, explain some of our cost-effectiveness findings, and we acknowledge this possibility. In addition, dividing the analysis into a subgroup led to the sample size becoming smaller. Secondly, during the course of the original RCT study, one or both clinics could possibly have integrated many of the recommended approaches of the stepped-care pathway into their practices, therefore decreasing the apparent impact of a stepped-care pathway. Thirdly, the effectiveness data was derived from a relatively short study (12-week trial), and we assumed the observed quality of life at 12-week would be maintained until one year. This assumption may be debatable. We tested the assumption by examining two other scenarios in which the treatment effect was assumed to vanish immediately at 12 -week and to reduce gradually to baseline values at 52-week. The analysis found little change in the cost-effectiveness results.

The fourth possible limitation is that implementation costs were not included in the economic analysis. There would be set-up costs for the stepped-care pathway, and excluding these costs would underestimate the total costs. However, since these additional 

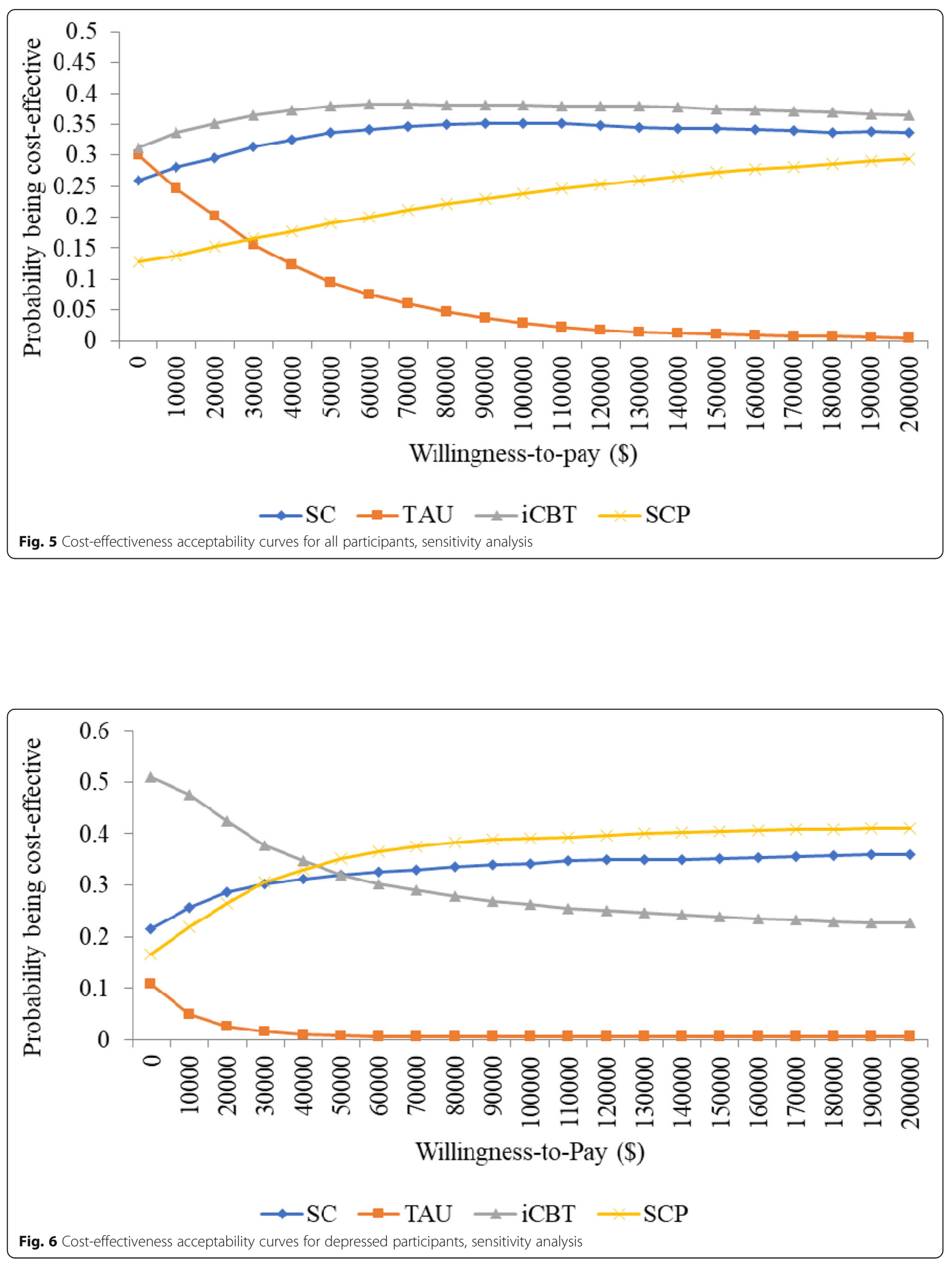
costs would occur only during the program implementation period, their effect on the intervention's overall cost-effectiveness is likely limited over the longer term. Finally, people with major depressive disorder experience higher rates of unemployment and other disabilities than their peers [6]. This aspect may have an impact on societal costs due to productivity losses and increasing use of care givers and/or social workers. However, because the analysis specifically focuses on the health care system, costs outside the scope were not considered. Gender difference in both health care usage and depression symptoms is also worth keeping in mind when interpreting our results. Female participants account for around $70 \%$ of total participants in our analysis. This proportion shows a similar trend suggested in the previous literature in which women had much higher rates of depressive disorder compared to men $[52,53]$. Our analysis did not split the resource use and costs by gender, as it was predicated on universal screening at a family practice and so is presumed to represent patients using the system. In addition, our results are for patients who go to an appointment at a primary care physician. So overall costs would be altered by the impact of people who do not see a doctor at all.

\section{Conclusion}

The present economic analysis finds that even where there are no clinically significant differences in health outcome, economic savings may arise from implementing the stepped-care model. As with previous studies, we found that individuals who had depression incurred greater health care costs than those who were not depressed. However, somewhat counter-intuitively, our results suggest that a more comprehensive stepped-care pathway may lead to significant savings in overall health care costs versus treatment as usual for the entire population, not only those who screened as depressed at baseline. These findings, if validated in other studies, could have major economic implications for health care systems. As stepped-care pathways have been increasingly adopted by clinicians and policy makers $[15,16,22]$, the economic impact of this treatment approach typically causes concern $[19,20]$. Our results, if supported by future research, would help address these issues and possibly assist health care planners in making more informed choices. While more work is required to identify the most clinically effective versions of a stepped-care pathway, our findings suggest that the care pathway may have substantial potential to improve health care system value in terms of a lower incremental cost-effectiveness ratio compared with treatment as usual and online-based cognitive behaviour therapy.

\section{Additional file}

Additional file 1: Table S1. Number of participants in the four treatment groups separated into depressed and non-depressed subgroups. (DOCX $207 \mathrm{~kb}$ )

\begin{abstract}
Abbreviations
CBT: Cognitive Behavioural Therapy; Cl: Confidence Interval; CPI: Consumer Price Index; EQ-5D-5 L: Eurogol-5-Dimension with five level scale; GLM: Generalized Linear Model; HRQoL: Health-Related Quality of Life; iCBT: Online Cognitive Behavioural Therapy; ICER: Incremental CostEffectiveness Ratio; IQR: Interquartile Range; ISPOR: International Society for Pharmacoeconomics and Outcomes Research; ITT: Intention-To-Treat; OLS: Ordinary Least Squares; PHQ-9: Patient Health Questionnaire - 9-Item; QALY: Quality-Adjusted Life-Year; RCT: Randomized, Controlled Trial; s.d.: Standard Deviation; SC: Standard Care; SCP: Stepped-Care Pathway; TAU: Treatment-as-Usual
\end{abstract}

\section{Acknowledgements}

This study was supported by the Addiction \& Mental Health Strategic Clinical Network, Alberta Health Services. We would like to thank external reviewers Luca Jansses, Gemma Shields and Research Square for comments on the manuscript; Christopher McCabe for comments on a previous draft and Jeff Round for comments on the revised version; and Deena Hamza, Stefanie Kletke, Tennile Tavares, and Michael Lahey for research assistance. We would also like to thank the Primary Care Networks that participated in the trial.

\section{Authors' contributions}

PS is the PI of the study, and he and KR have been involved in all aspects of the study design and implementation. CY contributed to the conceptualisation and design of the economic components. CY and SS are responsible for data analysis, data collection, as well as health economic analysis. All authors contributed to preparing and revising this manuscript. All authors read and approved the final manuscript and are accountable for all aspects of the accuracy and integrity of the manuscript.

\section{Funding}

Financial support for this study was provided entirely by a financial contribution from Alberta Health Services (AHS). The funding agreement ensured the authors' independence in designing the study, interpreting the data, writing, and publishing the study.

\section{Availability of data and materials}

We are part of the research team who has access to datasets of the study for publications. Governed by privacy legislation and agreements between the research team and provider agencies, the primary population health and economic data are not part of datasets available for other analyses.

\section{Ethics approval and consent to participate}

The clinical trial reported in the analysis has received written consent from the Health Research Ethics Review Board at the University of Alberta (Pro00038495) for adults aged 18 who were cognitively capable of giving informed consent. Approval was first given on 30th July 2013 and then included some small changes.

\section{Consent for publication}

Not applicable.

\section{Competing interests}

The authors declare that they have no competing interests.

\section{Author details}

${ }^{1}$ Institute of Health Economics, 1200 - 10405 Jasper Avenue, Edmonton, Alberta T5J 3N4, Canada. ${ }^{2}$ Department of Psychiatry, Addiction \& Mental Health Strategic Clinical Network, Alberta Health Services, University of Alberta, 10030107 St, NW, Edmonton, Alberta T5J 3E4, Canada. ${ }^{3}$ Cumming School of Medicine, University of Calgary, 3330 Hospital Drive NW, Calgary, Alberta T2N 4N1, Canada. ${ }^{4}$ Department of Psychiatry, University of Alberta, 8440112 St NW, Edmonton, Alberta T6G 2B7, Canada. 
Received: 7 September 2018 Accepted: 29 July 2019 Published online: 05 August 2019

\section{References}

1. Ferrari AJ, Charlson FJ, Norman RE, et al. Burden of depressive disorders by country, sex, age, and year: findings from the global burden of disease study 2010. PLoS Med. 2013;10:e1001547.

2. Murray CJ, Lopez AD. Global mortality, disability, and the contribution of risk factors: global burden of disease study. Lancet. 1997;349:1436-42

3. Lam RW, Mclntosh D, Wang J, et al. Canadian network for mood and anxiety treatments (CANMAT) 2016 clinical guidelines for the management of adults with major depressive disorder: section 1. Disease burden and principles of care. Can J Psychiatry. 2016;61:510-23.

4. Kessler RC, Bromet EJ, de Jonge P, et al. The burden of depressive illness. Public Health Perspect Depress Disord. 2017:40.

5. Craven MA, Bland R. Depression in primary care: current and future challenges. Can J Psychiatry. 2013;58:442-8.

6. Rizvi SJ, Cyriac A, Grima E, et al. Depression and employment status in primary and tertiary care settings. Can J Psychiatry. 2015;60:14-22.

7. Deneke DE, Schultz HE, Fluent TE. Screening for depression in the primary care population. Psychiatr Clin. 2015;38:23-43.

8. Thielke S, Vannoy S, Unützer J. Integrating mental health and primary care. Prim Care. 2007;34:571-92.

9. Sreeharan V, Madden $\mathrm{H}$, Lee JT, et al. Improving access to psychological therapies and antidepressant prescribing rates in England: a longitudinal time-series analysis. Br J Gen Pract. 2013;63:e649-e53.

10. Hargraves D, White C, Frederick R, et al. Implementing SBIRT (screening, brief intervention and referral to treatment) in primary care: lessons learned from a multi-practice evaluation portfolio. Public Health Rev. 2017;38:31.

11. Rost $T$, Stein J, Löbner $M$, et al. User acceptance of computerized cognitive behavioral therapy for depression: systematic review. J Med Internet Res. 2017;19.

12. Dorow M, Löbner M, Pabst A, et al. Preferences for depression treatment including internet-based interventions: results from a large sample of primary care patients. Front Psych. 2018;9.

13. Lin EH, VonKorff M, Russo J, et al. Can depression treatment in primary care reduce disability?: a stepped care approach. Arch Fam Med. 2000;9:1052

14. Seekles W, van Straten A, Beekman A, et al. Stepped care treatment for depression and anxiety in primary care a randomized controlled trial. Trials. 2011;12:171.

15. Oosterbaan DB, Verbraak MJ, Terluin B, et al. Collaborative stepped care v. care as usual for common mental disorders: 8-month, cluster randomised controlled trial. Br J Psych. 2013;203:132-9.

16. Farooq S. Collaborative care for depression: a literature review and a model for implementation in developing countries. Int Health. 2013;5:24-8.

17. van Straten A, Seekles W, van't Veer-Tazelaar NJ, et al. Stepped care for depression in primary care: what should be offered and how? Med J Aust. 2010;192:S36.

18. Menchetti M, Sighinolfi C, Di Michele V, et al. Effectiveness of collaborative care for depression in Italy. A randomized controlled trial. Gen Hosp Psychiatry. 2013;35:579-86.

19. Richards DA, Bower P, Pagel C, et al. Delivering stepped care: an analysis of implementation in routine practice. Implement Sci. 2012;7:3.

20. Silverstone PH, Rittenbach K, Suen VY, et al. Depression outcomes in adults attending family practice were not improved by screening, stepped-care, or online CBT during a 12-week study when compared to controls in a randomized trial. Front Psych. 2017;8.

21. Driot $D$, Bismuth $M$, Maurel $A$, et al. Management of first depression or generalized anxiety disorder episode in adults in primary care: a systematic metareview. Presse Med. 2017.

22. Quigley L, Dobson K, Pusch D, et al. Integrating mental health services into primary care: a clinical pathway for the treatment of depression. Psynopsis. 2012:4:26-7

23. Lakkis NA, Mahmassani DM. Screening instruments for depression in primary care: a concise review for clinicians. Postgrad Med. 2015;127:99-106.

24. Kroenke K, Spitzer RL. The PHQ-9: a new depression diagnostic and severity measure. Psychiatr Ann. 2002;32:509-15.

25. Kroenke K, Spitzer RL, Williams JB, et al. The patient health questionnaire somatic, anxiety, and depressive symptom scales: a systematic review. Gen Hosp Psychiatry. 2010;32:345-59.
26. Devlin NJ, Shah KK, Feng Y, et al. Valuing health-related quality of life: an EQ-5 D-5 L value set for E ngland. Health Econ. 2018;27:7-22.

27. Herdman M, Gudex C, Lloyd A, et al. Development and preliminary testing of the new five-level version of EQ-5D (EQ-5D-5L). Qual Life Res. 2011;20:1727-36.

28. EuroQol G. EuroQol--a new facility for the measurement of health-related quality of life. Health policy (Amsterdam, Netherlands). 1990; 16: 199.

29. Twomey C, O'reilly G, Byrne M, et al. A randomized controlled trial of the computerized CBT programme, MoodGYM, for public mental health service users waiting for interventions. Br J Clin Psychol. 2014;53:433-50.

30. Rabin R. Charro Fd. EQ-SD: a measure of health status from the EuroQol group. Ann Med. 2001;33:337-43.

31. Ramsey SD, Willke RJ, Glick H, et al. Cost-effectiveness analysis alongside clinical trials II-an ISPOR good research practices task force report. Value Health. 2015;18:161-72.

32. Wolowacz SE, Briggs A, Belozeroff $V_{\text {, }}$ et al. Estimating health-state utility for economic models in clinical studies: an ISPOR good research practices task force report. Value Health. 2016;19:704-19.

33. Montori VM, Guyatt GH. Intention-to-treat principle. Can Med Assoc J. 2001;165:1339-41.

34. Sterne JA, White IR, Carlin JB, et al. Multiple imputation for missing data in epidemiological and clinical research: potential and pitfalls. Bmj. 2009;338:b2393.

35. Wooldridge JM. Econometric analysis of cross section and panel data. Cambridge: MIT press; 2010.

36. Newhouse JP. Health economics and econometrics. Am Econ Rev. 1987:77: 269-74.

37. Basu A, Manning WG. Issues for the next generation of health care cost analyses. Med Care. 2009;47:S109-S14.

38. Briggs $\mathrm{A}$, Sculpher $\mathrm{M}$, Claxton $\mathrm{K}$. Decision modelling for health economic evaluation. OUP Oxford, 2006.

39. Drummond MF, Sculpher MJ, Claxton K, et al. Methods for the economic evaluation of health care programmes. Oxford: Oxford university press; 2015.

40. Glick HA, Doshi JA, Sonnad SS, et al. Economic evaluation in clinical trials. OUP Oxford, 2014

41. Briggs $\mathrm{AH}$, Mooney $\mathrm{CZ}$, Wonderling DE. Constructing confidence intervals for cost-effectiveness ratios: an evaluation of parametric and non-parametric techniques using Monte Carlo simulation. Stat Med. 1999:18:3245-62.

42. O'brien BJ, Drummond MF, Labelle RJ, et al. In search of power and significance: issues in the design and analysis of stochastic costeffectiveness studies in health care. Med Care. 1994:150-63.

43. Briggs $\mathrm{AH}$. Handling uncertainty in cost-effectiveness models. Pharmacoeconomics. 2000;17:479-500.

44. Scuffham PA, Yelland MJ, Nikles J, et al. ARE N-of-1 trials an economically viable option to improve access to selected high cost medications? The Australian Experience. Value Health. 2008;11:97-109.

45. Neumann PJ, Cohen JT, Weinstein MC. Updating cost-effectiveness-the curious resilience of the \$50,000-per-QALY threshold. N Engl J Med. 2014;371:796-7.

46. Grosse SD. Assessing cost-effectiveness in healthcare: history of the $\$ 50,000$ per QALY threshold. Expert Rev Pharmacoecon Outcomes Res. 2008:8:165-78.

47. Cohen J, Neumann PJ, Weinstein MC. Does preventive care save money? Health economics and the presidential candidates. N Engl J Med. 2008;358:661-3.

48. Richards DA, Ekers D, McMillan D, et al. Cost and outcome of Behavioural activation versus cognitive Behavioural therapy for depression (COBRA): a randomised, controlled, non-inferiority trial. Lancet. 2016;388:871-80.

49. Gensichen J, Petersen JJ, Von Korff M, et al. Cost-effectiveness of depression case management in small practices. Br J Psychiatry. 2013;202:441-6.

50. Mukuria C, Brazier J, Barkham M, et al. Cost-effectiveness of an improving access to psychological therapies service. Br J Psychiatry. 2013;202:220-7.

51. Grochtdreis T, Brettschneider C, Wegener A, et al. Cost-effectiveness of collaborative care for the treatment of depressive disorders in primary care: a systematic review. PLoS One. 2015;10:e0123078.

52. Salk RH, Hyde JS, Abramson LY. Gender differences in depression in representative national samples: meta-analyses of diagnoses and symptoms. Psychol Bull. 2017;143:783.

53. Luppa M, Sikorski C, Luck T, et al. Age-and gender-specific prevalence of depression in latest-life-systematic review and meta-analysis. J Affect Disord. 2012;136:212-21

\section{Publisher's Note}

Springer Nature remains neutral with regard to jurisdictional claims in published maps and institutional affiliations. 